\title{
Post-COVID 19 Neurological Syndrome: a fresh challenge in neurological management
}

\author{
Mauro Antonio González-Herazo ${ }^{1}$, Diana Carolina Silva-Muñoz ${ }^{2}$, Paula Andrea Guevara-Martínez ${ }^{3}$, \\ Ivan David Lozada-Martinez ${ }^{1}$ \\ ${ }^{1}$ University of Cartagena, Cartagena, Colombia \\ ${ }^{2}$ University of Santander, Colombia \\ ${ }^{3}$ University Foundation of Health Sciences, Colombia
}

Key words: Post-COVID 19 Neurological Syndrome, COVID-19, nervous system diseases, prognosis

(Neurol Neurochir Pol 2021; 55 (4): 413-414)

\section{To the Editors}

We read with great interest the article published recently by Zielińska-Turek et al. [1] entitled 'Clinical features of neurological patients with coronavirus 2019: an observational study of one centre', where the authors investigated a cohort of patients with COVID-19 and previous neurological diseases, finding that most of these patients were elderly, with dementia or a history of stroke, and that these conditions associated with COVID-19 worsened the prognosis and increased the mortality of these patients.

We thank Zielińska-Turek et al. [1] for providing such valuable evidence. However, we would like to make some comments on a recently described entity which poses a challenge in the management of patients with a neurological history or who present with neurological complications during the course of COVID-19.

Disease burden studies of neurological disorders estimate that since 2005 there has been a notable increase in Years Lived with Disability (YLDs) and Disability Adjusted Life Years (DALYs) globally, mainly due to cerebrovascular diseases (50,785 to 53,815 DALYs) and Alzheimer's and other types of dementia (11,078 to 13,540 DALYs) [2], and these figures are projected to increase to 60,864 and 18,394 by 2030 , respectively [2].

Based on the above, controlling the burden of neurological diseases is a global health priority.

Considering that the moderate and severe phenotypes of COVID-19 generally present neurological complications, or manifest themselves only through neurological syndromes [3], it is necessary to establish the prognosis of these patients and the impact on their quality of life.

Post-COVID 19 neurological syndrome is a new condition only recently described $[4,5]$, which consists of medium and long-term involvement of the nervous system due to molecular mechanisms that trigger neuroinflammation, compromising the functional capacity and quality of life of neurological patients in general $[4,5]$.

Noting that the average age of the patients of Zielińska-Turek et al. [1] was 72 years, and that they also had comorbidities that have a negative impact on the nervous system such as arterial hypertension, type II diabetes mellitus, or dementia [1], it is necessary to pay attention and perform strict follow-up of these patients, because of the increased probability and severity of developing post-COVID-19 neurological syndrome, manifested through the persistence of certain symptoms, or the presentation of neurological disorders in a short period after the resolution of COVID-19 $[4,5]$.

There is no clear evidence or understanding of the pathophysiology of this syndrome, although meta-analyses have reported results establishing short-term associations between COVID-19 and neurological involvement in patients presenting with neurological symptoms, in the absence of lesions visible on neuroimaging, regardless of age or expression of the COVID-19 phenotype [3].

In this scenario, a strategic plan should be designed by neurology and neurosurgery departments with the aim of creating prospective multicentre studies to evaluate the 
evolution of neurological involvement and rehabilitation of these patients, in order to establish effective and safe measures to ensure functional capacity and survival.

Likewise, this event sheds further light on the need to create specialised centres in neurointervention and neurorehabilitation, since the burden of neurological diseases remains one of the highest globally, and can be expected to increase even more rapidly due to this syndrome.

\section{References}

1. Zielińska-Turek J, Jasińska A, Kołakowska J, et al. Clinical features of neurological patients with coronavirus 2019: an observational study of one centre. Neurol Neurochir Pol. 2021; 55(2): 195-201, doi: 10.5603/PJNNS.a2021.0011, indexed in Pubmed: 33528832.
2. World Health Organization. Global burden of neurological disorders: estimates and projections. https://www.who.int/mental_health/neurology/ chapter_2_neuro_disorders_public_h_challenges.pdf (21 May 2021).

3. Rogers JP, Chesney E, Oliver D, et al. Psychiatric and neuropsychiatric presentations associated with severe coronavirus infections: a systematic review and meta-analysis with comparison to the COVID-19 pandemic. Lancet Psychiatry. 2020; 7(7): 611-627, doi: 10.1016/ S2215-0366(20)30203-0, indexed in Pubmed: 32437679.

4. Camargo-Martínez W, Lozada-Martínez I, Escobar-Collazos A, et al. Post-COVID 19 neurological syndrome: Implications for sequelae's treatment. J Clin Neurosci. 2021; 88: 219-225, doi: 10.1016/j. jocn.2021.04.001, indexed in Pubmed: 33992187.

5. Wijeratne T, Crewther S. Post-COVID 19 Neurological Syndrome (PCNS); a novel syndrome with challenges for the global neurology community. J Neurol Sci. 2020; 419: 117179, doi: 10.1016/j. jns.2020.117179, indexed in Pubmed: 33070003. 